\title{
SESAME Equation of State for Epoxy
}

\section{J. C. Boettger}




\title{
SESAME Equation of State For Epoxy
}

by

\author{
J. C. Boettger
}

\begin{abstract}
A new SESAME equation of state (EOS) for epoxy has been generated using the computer program GRIZZLY. This new EOS has been added to the SESAME EOS library as material number 7603.
\end{abstract}

\section{INTRODUCTION}

Users of the SESAME equation of state (EOS) library' have a long standing interest in the EOSs of polymers and polymer composites. In response to this interest, there has been an ongoing effort to expand the SESAME library to include EOSs for additional polymers and to upgrade the quality of existing polymer EOSs as new experimental data becomes available. One of the most widely used polymers is epoxy, a prototypical thermoset polymer. Currently, the SESAME library includes two EOSs for epoxy, material numbers 7601 and 7602 , both added to the library in 1984 . (These two EOSs are identical, except 7601 has van der Waals loops in the vapor dome region, whereas 7602 has Maxwell constructions.) Like many of the older EOSs in the SESAME library, these existing EOSs for epoxy are largely undocumented and it is 
difficult to tell exactly what type of epoxy is being represented. In addition, the old EOSs for epoxy have a bulk sound speed $(2.75 \mathrm{~km} / \mathrm{s})$ that is significantly larger than the experimental value $(2.263 \mathrm{~km} / \mathrm{s})^{2}$

Recently, a number of experiments were conducted on a specific type of epoxy formed by mixing Epon 828 resin (70\% by weight) with Jeffamine T-403 curing agent ( $30 \%$ by weight). ${ }^{3}$ First, the elemental compositions of the two components used to form the epoxy were carefully determined. ${ }^{3}$ Then, the density and bulk sound speed of the cured epoxy were measured; yielding $1.154 \mathrm{gm} / \mathrm{cm}^{3}$ and $2.255 \mathrm{~km} / \mathrm{s}$, respectively. ${ }^{4}$ Finally, a series of shock wave experiments was used to determine a number of points along the principal Hugoniot of the cured epoxy. ${ }^{4}$

In the present investigation, the new experimental data for epoxy $y^{3,4}$ have been used, in conjunction with older Hugoniot data, ${ }^{2}$ to construct a new SESAME EOS for epoxy. This new EOS for epoxy is not subject to the difficulties associated with the older EOSs, discussed above. The new EOS will be added to the SESAME library as material number 7603 .

\section{METHODOLOGY}

For most of the EOSs in the SESAME library, the pressure $P$, internal energy $E$, and Helmholtz free energy $A$ are all partitioned into three distinct terms:

$$
P(\rho, T)=P_{s}(\rho)+P_{n}(\rho, T)+P_{c}(\rho, T)
$$




$$
\begin{aligned}
& E(\rho, T)=E_{s}(\rho)+E_{n}(\rho, T)+E_{e}(\rho, T) \\
& A(\rho, T)=A_{s}(\rho)+A_{n}(\rho, T)+A_{c}(\rho, T)
\end{aligned}
$$

where $\rho$ is the density and $T$ is the temperature. The three subscripts $s, n$, and $e$ denote the contributions to the EOS from the static lattice cold curve (zero temperature isotherm), the nuclear motion, and the thermal electronic excitations, respectively. Thus, it is possible to calculate (or update) each term independently using any desired model. Here, all three pieces of the EOS for epoxy have been generaled with GRIZZLY, ${ }^{5}$ the locally developed multipurpose computer program for calculating SESAME EOSs.

In GRIZZLY, the only model currently available for calculating the thermal electronic contributions is the Thomas-Fermi-Dirac (TFD) model ${ }^{6}$ For the present EOS, thermal electronic contributions were first generated for each atomic component in epoxy. Those monatomic thermal electronic EOSs were then combined via additive volume mixing. ${ }^{5}$ This part of the calculation requires the weight fraction $\left(w_{i}\right)$, atomic number $\left(Z_{i}\right)$, and atomic mass $\left(A_{i}\right)$ for each species of atom (i) in the epoxy. As was noted above, the epoxy was formed from a mixture of Epon 828 resin $(70 \%$ by weight) and Jeffamine T-403 curing agent ( $30 \%$ by weight). ${ }^{3}$ Table 1 gives the atomic compositions measured for the two constituents of the epoxy, ${ }^{3}$ neglecting the trace clements. Table 1 also gives the atomic composition used here for the cured epoxy (based on the assumption that the composition is not altered by the curing process) and the values of $A_{i}$ and $Z_{i}$ used for each atom. ${ }^{7}$ 
Table 1: Atomic Compositions Of

Epon 828, Jeffamine T-403, and Epoxy

\begin{tabular}{|l|c|c|c|c|c|}
\hline Atom & $Z_{i}$ & $A_{i}$ & \multicolumn{3}{|c|}{$W_{i}$} \\
\hline & & & Epon & T-40.3 & Epoxy \\
\hline & & & & & \\
$\mathrm{C}$ & 6.0 & 12.01 & 0.741 & 0.599 & 0.6984 \\
$\mathrm{O}$ & 8.0 & 15.999 & 0.188 & 0.193 & 0.1895 \\
$\mathrm{H}$ & 1.0 & 1.008 & 0.071 & 0.112 & 0.0833 \\
$\mathrm{~N}$ & 7.0 & 14.007 & & 0.096 & 0.0288 \\
& & & & & \\
\hline
\end{tabular}

The thermal nuclear contributions for epoxy were obtained with the JDJNUC nuclear model ${ }^{8}$ in GRIZZLY. In this model, the material is treated as a Debye solid at low temperatures and as an ideal gas at high temperatures. The JDJNUC model smoothly switches between these two limiting forms for temperatures near the melting line, which is obtained from the Lindemann law. On each nuclear isotherm, the melting transition is approximated by a small two phase region located at the melting point.

In addition to the data listed in Table 1 and the ambient density $\left(\rho_{0}=1.154\right.$ $\left.\mathrm{gm} / \mathrm{cm}^{3}\right),{ }^{4}$ the JDJNUC model requires an ambient melt temperature $\left(T_{m}\right)$ and some analytical form for the Gruneisen parameter as a function of density $[\gamma(\rho)]$. The Debye temperature $\left(\Theta_{D}\right)$ is calculated internally from $T_{m}$ via an empirical relationship based on the behavior of pure elements. ${ }^{8}$ Because epoxy is a thermoset polymer, it does not have a well-defined melting temperature to be used in GRIZZLY. Here, $T_{m}=1000 \mathrm{~K}$ has been chosen as a reasonable estimate. The associated Debye temperature then is $\Theta_{D}=843 \mathrm{~K}$ 
A generalized version of the standard CHART-D model ${ }^{9}$ has been used to describe $\gamma(p)$; the IGRUN $=7$ option in GRIZZLY. ${ }^{5}$ In this formulation, the Gruneisen parameter is expressed as a quadratic function of $\rho$ in the expanded region and as a quadratic function of $1 / \rho$ in the compressed region. Four items of input are required to completely specify $\gamma(\rho)$ : the ambient Gruneisen parameter $\left(\gamma_{0}\right)$, the ambient value of the logarithmic derivative of $\gamma$, and the asymptotic values $\gamma(0)$ and $\gamma(\infty)$. The ambient Gruneisen parameter was chosen to match the value used in the two existing SESAME EOSs for epoxy, $\gamma_{0}=0.8 .{ }^{10}$ (This value for $\gamma_{0}$ is also consistent with the recent shock wave experiments. ${ }^{4}$ ) The logarithmic derivative at $\rho_{0}$ was chosen to have a small negative value $(-0.05)$ to ensure sensibly smooth nuclear isotherms near $\rho_{0}$. For $\gamma(0)$ and $\gamma(\infty)$ the default values of 1 and $2 / 3$ were used.

For modest compressions $(\leq 2.0)$, the cold curve was obtained by removing thermal contributions from an input Hugoniot read in as a table of particle and shock velncities $\left(U_{p}, U_{s}\right)$ selected to provide a good fit to all available Hugoniot and sound speed data; ${ }^{2,4}$ see Table 2 . For high compressions, the cold curve was required to smoothly extrapolate to a mixed TFD cold curve. This method of calculation ensures that the compressed EOS will reproduce the experimental Hugoniot and also will have the correct asymptotic behavior. In the expanded region $\left(\rho<\rho_{0}\right)$, the cold curve was fitted with a generalized Lennard-Jones form ${ }^{5}$ that was constrained to smoothly connect with the compressed portion of the cold curve and have the correct cohesive energy $\left(E_{c}\right)$. Here, $E_{c}=40 \mathrm{kcal} / \mathrm{av}$-atom-mol was chosen to ensure that the final EOS has a reasonable vapor dome. In addition to the parameters already discussed, this part 
of the calculation requires a parameter FACLJ ${ }^{5}$ (here 0.5 ) that controls the shape of the expanded cold curve.

Table 2: Input Hugoniot Points

\begin{tabular}{|c|c|c|r|}
\hline$U_{p}(\mathrm{~km} / \mathrm{s})$ & $U_{s}(\mathrm{~km} / \mathrm{s})$ & $U_{p}(\mathrm{~km} / \mathrm{s})$ & $U_{s}(\mathrm{~km} / \mathrm{s})$ \\
\hline & & & \\
0.000 & 2.263 & 2.995 & 7.195 \\
0.185 & 2.865 & 3.215 & 7.195 \\
0.370 & 3.250 & 5.500 & 10.237 \\
\hline
\end{tabular}

\section{RESULTS}

Figure 1 compares the present theoretical Hugoniot for epoxy with the experimental data from Ref. 2 (diamonds) and Ref. 4 (stars). (The overall agreement between the old data ${ }^{2}$ and the new data ${ }^{4}$ is reassuring.) The theoretical Hugoniot for material number 7601 is also shown in Fig. 1. There are two significant features apparent hoth in the experimental data and in the Hugoniot for material number 7603 . First, there is a phase transition in the region $2.995<U_{p}<3.215 \mathrm{~km} / \mathrm{s}$. This phase transition, which also appears in the Hugoniot for material number 7601 , is a standard feature of polymers and may be due to pressure induced crosslinking between the chains of the polymer."

The second important feature in Fig. 1 is the rapid drop in the Hugoniot shock velocity as the particle velocity approaches zero. Again, this is a rather common feature of polymers and may be due to the large difference between the interchain and 
intrachain binding. "At ambient conditions, the elastic properties of the polymer are dominated by the weak interchain binding, producing a rather small bulk modulus and sound speed. As the interchain separation is reduced under pressure, the bulk modulus and $U_{s}$ both increase rapidly until the interchain spacing becomes comparable to the intrachain spacing, at which point the Hugoniot becomes linear. The new EOS for epoxy clearly provides a good fit to all of the experimental data in Fig. 1. In contrast, the theoretical Hugoniot for material number 7601 fails to match the experimental sound speed data.

Although the current EOS for epoxy is similar to material number 7601 , the new SESAME EOS for epoxy should provide a better description of cured epoxy near ambient conditions. The new EOS should be very reliable for a shock loading, or other processes that stay close to the principal Hugoniot. Material number 7603 will be added to the SESAME library in the near future. 


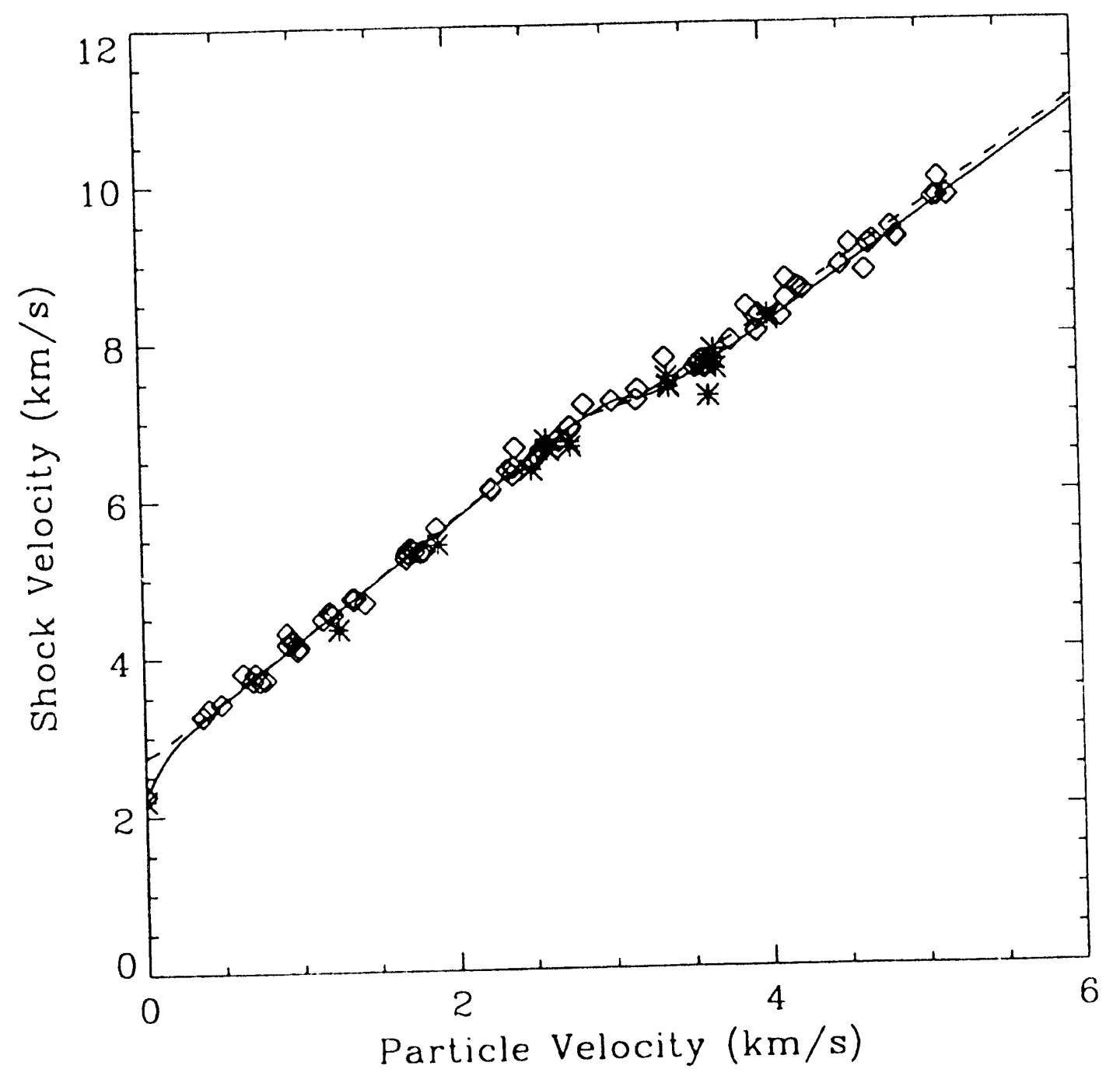

Figure 1. The current theoretical Hugoniot for epoxy (solid line) is compared with the theoretical Hugoniot for material number 7601 (dashed line) and with experimental Hugoniot and sound speed data from Ret. 2 (diamonds) and Ref. 4 (stars). 


\section{REFERENCES}

1. S. P. Lyon and J. D. Johnson, editors, "SESAME: The Los Alamos National Laboratory Equation Of State Database," Los Alamos National Laboratory Reporl LA-UR-92-3407 (1992).

2. S. P. Marsh, editor, LASL Shock Hugoniot Data (U. C. Press, Berkeley, CA, 1980).

3. C. Sandoval, Los Alamos National Laboratory, personal communication (April. 1993).

4. B. Olinger, J. Fritz, and C. E. Morris, "Equations Of State For PEEK, Epon 828 , and Carbon Fiber - Epon Composite," unpublished preliminary report, Los Alamos National Laboratory (March, 1993).

5. Joseph Abdallah, Jr., "User's Manual for GRIZZLY," Los Alamos National Laboratory report LA-10244-M (September 1984).

6. R. D. Cowan and J. Ashkin, Phys. Rev. 105, 144 (1957).

7. N. W. Ashcroft and N. D. Mermin, Solid State Physics (Holt, Rinehart, and Winston, New York, 1976).

8. J. D. Johnson, "A Generic Model For The Ionic Contribution To The Equation (I) State," Los Alamos National Laboratory Report LA-12003-MS (1991).

9. S. L. Thompson and H. S. Lawson, "Improvements to the CHART-D RadiationHydrodynamics Code MI; Revised Analytic Equations of State," Sandia Laboratories report SC-RR-710714 (1972). 
10. J. D. Johnson, Los Alamos National Laboratory, personal communication (December. 1993).

11. W. J. Carter, S. P. Marsh, and R. G. McQueen, "Hugoniot Equation of State of Polymers," Los Alamos Scientific Laboratory Report LA-UR 77-2062 (1977). 

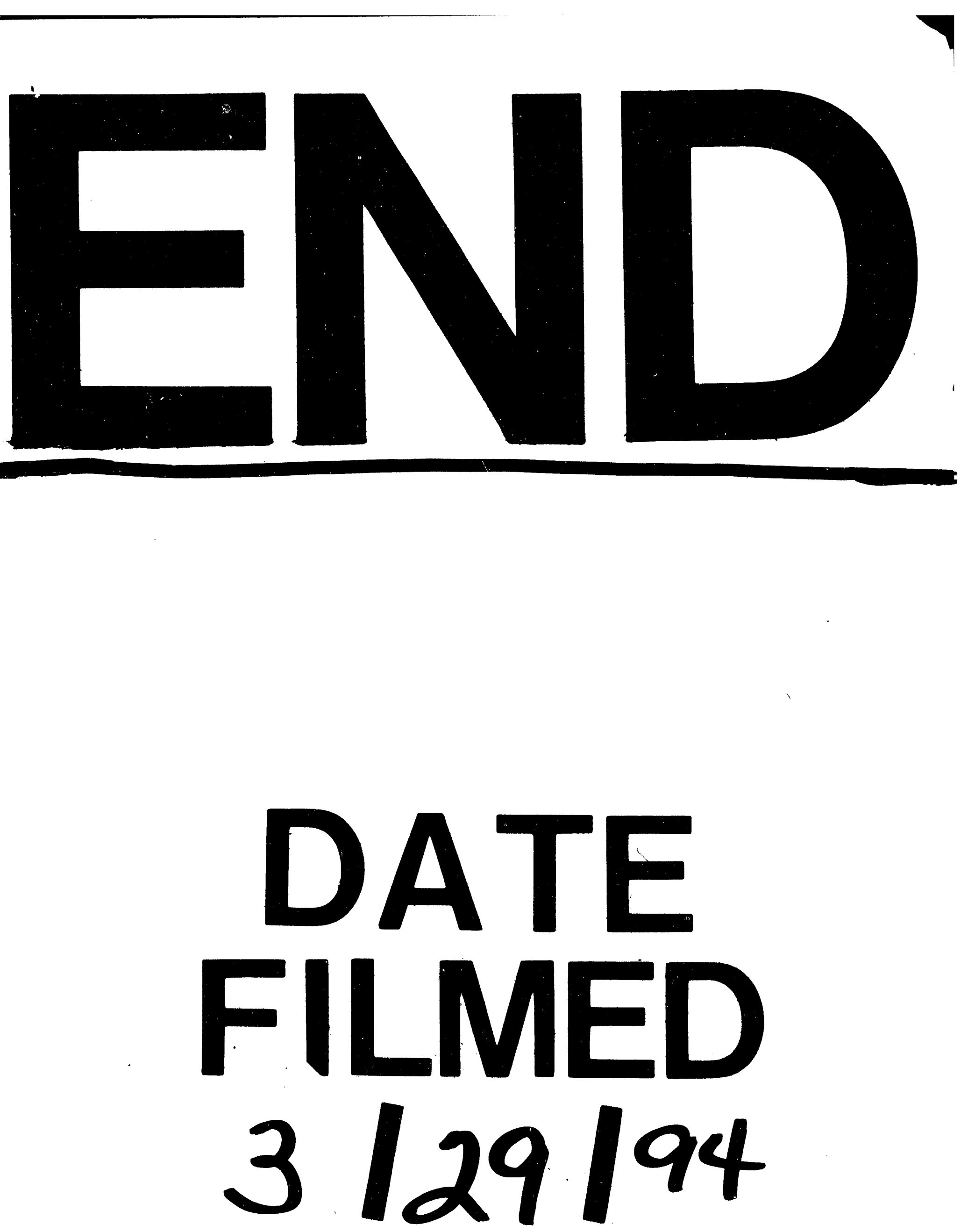